\title{
Characterizations of geodesic sub-b-s-convex functions on Riemannian manifolds
}

\author{
Izhar Ahmad ${ }^{\mathrm{a}, *}$, Anurag Jayswal ${ }^{\mathrm{b}}$, Babli Kumarib \\ ${ }^{a}$ Department of Mathematics and Statistics, King Fahd University of Petroleum and Minerals, Dhahran 31261, Saudi Arabia. \\ ${ }^{b}$ Department of Applied Mathematics, Indian Institute of Technology (Indian School of Mines), Dhanbad-826004, Jharkhand, India.
}

Communicated by N. Shahzad

\begin{abstract}
In this paper, we present the notion of geodesic sub-b-s-convex function on the Riemannian manifolds. A non-trivial example of geodesic sub-b-s-convex function but not geodesic convex function is also discussed. Some fundamental properties of geodesic sub-b-s-convex functions are investigated. Moreover, we derive the optimality conditions of unconstrained and constrained programming problems under the sub-b-s-convexity.
\end{abstract}

Keywords: Geodesic convex set, geodesic sub-b-s-convex function, optimality conditions, Riemannian manifolds. 2010 MSC: 26A51, 53C22, 58B20, 90C46.

(C)2018 All rights reserved.

\section{Introduction}

Convexity plays a significant part in numerous disciplines such as mathematics, management science, economics, engineering, and various applied sciences. It is mostly vital to the analysis of optimization problems where convexity is determined by different appropriate properties. However, so far all real-life problems can not be described by a convex mathematical model. That's why we generalized convex function because it gives more accurate results of reality in many cases. Hanson [6] introduced a new concept of invexity as a generalization of convexity. For more information on generalized convex functions, see $[3,5,7,11,15]$.

On the other hand, geodesic convex function is a natural generalization of convex function on Riemannian manifolds proposed by Rapcsak [13] and Udriste [14]. In these work, a line segment is replaced by a geodesic and a linear space is replaced by a Riemannian manifold. Further, Ahmad et al. [2] , Iqbal et al. $[8,10]$, and Agarwal et al. [1] presented the notions of geodesic $\eta$-preinvex, strong geodesic $\alpha$-preinvexity,

\footnotetext{
*Corresponding author

Email addresses: drizhar@kfupm.edu.sa (Izhar Ahmad), anurag_jais123@yahoo.com (Anurag Jayswal), bablichoudhary69@yahoo. com (Babli Kumari)
}

doi: 10.22436/jnsa.011.02.02 
geodesic E-convex sets and preinvex functions on Riemannian manifolds. Many authors studied the various results of generalized convexity on Riemannian manifolds (see, for example $[4,9,10,16]$ ).

Incentive by the work of [12], we extend these results on Riemannian manifolds under the sub-bs-convexity. The content of this paper is arranged as follows. In Section 2, we present some known notations and definitions, which will help us to study this paper. In Section 3, a new class of functions and sets namely, geodesic sub-b-s-convex function and geodesic sub-b-s-convex set are introduced. Several properties of these functions are also discussed. We study the optimality conditions of unconstrained and constrained programming problems under the sub-b-s-convexity in Section 4 . Finally, we present our conclusion in Section 5.

\section{Preliminaries}

Let $\mathrm{N}$ be a finite dimensional Riemannian manifold and $\mathrm{D} \subseteq \mathrm{N}$ be a non-empty set with a Riemannian metric $\langle.,$.$\rangle on the \mathrm{T}_{v} \mathrm{~N}$, where $\mathrm{T}_{v} \mathrm{~N}$ denotes the tangent space of $\mathrm{N}$ at $v$ for $v \in \mathrm{N}$. The associated norm is denoted by $\|\cdot\|_{v}$, where the subscript $v$ is sometimes skipped. Suppose $T N=\bigcup_{v \in N} T_{v} N$ is tangent bundle of $N$. Consider $u$ and $v$ are points in Riemannian manifold $N$. We denote the geodesic joining $u \in N$ and $v \in \mathrm{N}$ by $\gamma_{u v}:[0,1] \rightarrow \mathrm{N}$ such that $\gamma_{u v}(0)=v$ to $\gamma_{u v}(1)=u$.

Definition 2.1 ([14]). A subset D of $\mathrm{N}$ is called geodesic convex set, if D contains every geodesic $\gamma_{u v}$ of $\mathrm{N}$ whose end points $u$ and $v$ belong to $\mathrm{D}$.

Definition 2.2 ([14]). Let $\mathrm{D}$ be geodesic convex set in $\mathrm{N}$. A function $\psi: \mathrm{D} \rightarrow \mathbb{R}$ is called geodesic convex if

$$
\psi\left(\gamma_{u v}(t)\right) \leqslant t \psi(u)+(1-t) \psi(v)
$$

for every geodesic $\gamma_{u v}:[0,1] \rightarrow D, \gamma_{u v}(0)=v, \gamma_{u v}(1)=u$ for all $t \in[0,1]$ and $u, v \in D$.

\section{Geodesic sub-b-s-convex functions and properties}

We present the concept of geodesic sub-b-s-convex function on $\mathrm{D}$ with respect to $\mathrm{b}$ which is generalization of a function defined by Liao and $\mathrm{Du}[12]$.

Definition 3.1. A function $\psi: D \rightarrow \mathbb{R}$ is said to be geodesic sub-b-s-convex function with respect to map $\mathrm{b}: \mathrm{D} \times \mathrm{D} \times[0,1] \rightarrow \mathbb{R}$ on the geodesic convex set $\mathrm{D} \subset \mathrm{N}$, if

$$
\psi\left(\gamma_{u v}(t)\right) \leqslant t^{s} \psi(u)+(1-t)^{s} \psi(v)+b(u, v, t)
$$

holds for each $u, v \in D, t \in[0,1]$ and for some fixed $s \in(0,1]$.

Remark 3.2. When $s=1$ and $b(u, v, t)=0$, then geodesic sub-b-s-convex function reduces to the geodesic convex function.

Example 3.3. Let $N=\left\{e^{i \theta}: 0<\theta<\frac{\pi}{2}\right\}$ and $\psi: N \rightarrow \mathbb{R}$ be a function defined as $\psi\left(e^{i \theta}\right)=\cos \theta$ with $u, v \in N, u=e^{i \alpha}$ and $v=e^{i \beta}$. Suppose $\gamma_{u v}(t)=(\cos ((1-t) \beta+t \alpha), \sin ((1-t) \beta+t \alpha))$ and $b(u, v, t)=\frac{1}{t}$, where $b: N \times N \times[0,1] \rightarrow \mathbb{R}$. Then, $\psi\left(\gamma_{u v}(t)\right)=\cos ((1-t) \beta+t \alpha), \psi(u)=\psi\left(e^{i \alpha}\right)=\cos \alpha$, and $\psi(v)=\psi\left(e^{i \beta}\right)=\cos \beta$. Now we shall show that function $\psi$ is a geodesic sub-b-s-convex function for all $u, v \in \mathrm{N}, \mathrm{t} \in[0,1]$ and for some fixed $s=0.1$, because

$$
\begin{aligned}
& \psi\left(\gamma_{u v}(t)\right)-t^{s} \psi(u)-(1-t)^{s} \psi(v)-b(u, v, t) \\
& \quad=\cos ((1-t) \beta+t \alpha)-t^{0.1} \cos \alpha-(1-t)^{0.1} \cos \beta-\frac{1}{t} \leqslant 0 .
\end{aligned}
$$

But it is not geodesic convex function when $s=1$ and $b(u, v, t)=0$ at $t=\frac{1}{2}, \alpha=\frac{\pi}{4}$, and $\beta=\frac{\pi}{6}$. Clearly,

$$
\psi\left(\gamma_{u v}(t)\right)-t \psi(u)-(1-t) \psi(v)=\cos ((1-t) \beta+t \alpha)-t \cos \alpha-(1-t) \cos \beta=0.0068 \nless 0 .
$$


Now, we discuss several basic properties of geodesic sub-b-s-convex functions as follows.

Theorem 3.4. Let $\psi: \mathrm{D} \rightarrow \mathbb{R}$ be a geodesic sub-b-s-convex function with respect to $\mathrm{b}$ on the geodesic convex set $\mathrm{D}$ and $\Phi: \mathbb{R} \rightarrow \mathbb{R}$ be an increasing function. Then $\Phi$ o $\psi$ is a geodesic sub-b-s-convex function with respect to $\Phi$ o b.

Proof. Since $\psi$ is a geodesic sub-b-s-convex function with respect to $b$, we have

$$
\psi\left(\gamma_{u v}(t)\right) \leqslant t^{s} \psi(u)+(1-t)^{s} \psi(v)+b(u, v, t)
$$

holds for each $u, v \in D, t \in[0,1]$ and for some fixed $s \in(0,1]$. As $\Phi$ is an increasing function, it becomes

$$
\begin{aligned}
\Phi\left(\psi\left(\gamma_{u v}(t)\right)\right) & \leqslant \Phi\left(t^{s} \psi(u)+(1-t)^{s} \psi(v)+b(u, v, t)\right), \\
\Phi\left(\psi\left(\gamma_{u v}(t)\right)\right) & \leqslant t^{s} \Phi(\psi(u))+(1-t)^{s} \Phi(\psi(v))+\Phi(b(u, v, t)), \\
(\Phi \circ \psi)\left(\gamma_{u v}(t)\right) & \leqslant t^{s}(\Phi \circ \psi)(u)+(1-t)^{s}(\Phi \circ \psi)(v)+(\Phi \text { o b })(u, v, t) .
\end{aligned}
$$

Hence this completes the proof.

Theorem 3.5. If $\psi_{j}, j=1, \ldots, m$ are geodesic sub-b-s-convex functions with respect to $b_{j}, j=1, \ldots, m$ on $D$ and $a_{j} \geqslant 0, j=1, \ldots, m$, then $\psi=\sum_{j} a_{j} \psi_{j}$ is a geodesic sub-b-s-convex function with respect to $b=\sum_{j} a_{j} b_{j}$ on $D$.

Proof. By the hypothesis, we have

$$
\psi_{j}\left(\gamma_{u v}(t)\right) \leqslant t^{s} \psi_{j}(u)+(1-t)^{s} \psi_{j}(v)+b_{j}(u, v, t)
$$

holds for any $u, v \in D, t \in[0,1]$ and for some fixed $s \in(0,1]$. It follows that

$$
\begin{gathered}
a_{j} \psi_{j}\left(\gamma_{u v}(t)\right) \leqslant t^{s} a_{j} \psi_{j}(u)+(1-t)^{s} a_{j} \psi_{j}(v)+a_{j} b_{j}(u, v, t), \\
\sum_{j} a_{j} \psi_{j}\left(\gamma_{u v}(t)\right) \leqslant t^{s} \sum_{j} a_{j} \psi_{j}(u)+(1-t)^{s} \sum_{j} a_{j} \psi_{j}(v)+\sum_{j} a_{j} b_{j}(u, v, t) .
\end{gathered}
$$

Hence the theorem holds.

Theorem 3.6. If $\psi_{j}: D \rightarrow \mathbb{R}, j=1, \ldots, m$ are geodesic sub-b-s-convex functions with respect to $b_{j}, j=1, \ldots, m$, then $\psi=\max \left\{\psi_{j}, j=1, \ldots, m\right\}$ is a geodesic sub-b-s-convex function with respect to $b=\max \left\{b_{j}, j=1, \ldots, m\right\}$.

Proof. For each $u, v \in D, t \in[0,1]$ and for some fixed $s \in(0,1]$, according to the geodesic sub-b-s-convex functions $\psi_{j}$ with respect to $b_{j}$, we obtain

$$
\begin{aligned}
\psi_{j}\left(\gamma_{u v}(t)\right) & \leqslant t^{s} \psi_{j}(u)+(1-t)^{s} \psi_{j}(v)+b_{j}(u, v, t), \\
\max \left\{\psi_{j}\left(\gamma_{u v}(t)\right)\right\} & \leqslant \max \left\{t^{s} \psi_{j}(u)+(1-t)^{s} \psi_{j}(v)+b_{j}(u, v, t)\right\} \\
& =t^{s} \max \left\{\psi_{j}\right\}(u)+(1-t)^{s} \max \left\{\psi_{j}\right\}(v)+\max \left\{b_{j}\right\}(u, v, t) \\
& =t^{s} \psi(u)+(1-t)^{s} \psi(v)+b(u, v, t),
\end{aligned}
$$

or

$$
\psi\left(\gamma_{u v}(t)\right) \leqslant t^{s} \psi(u)+(1-t)^{s} \psi(v)+b(u, v, t) .
$$

Hence, $\psi$ is a geodesic sub-b-s-convex function with respect to $b$.

In the following, we introduce a new notion of set, which is a geodesic sub-b-s-convex set and we study some properties.

Definition 3.7. Let $U \subset N \times \mathbb{R}$. $U$ is said to be geodesic sub-b-s-convex set with respect to $b: D \times D \times$ $[0,1] \rightarrow \mathbb{R}$. Then for each pair of $(u, \alpha)$ and $(\nu, \beta) \in U$,

$$
\left(\gamma_{u v}(t),(1-t)^{s} \beta+t^{s} \alpha+b(u, v, t)\right) \in U
$$

for all $t \in[0,1]$ and for some fixed $s \in(0,1]$. 
Now, we characterize a geodesic sub-b-s-convex function $\psi: D \rightarrow \mathbb{R}$ in term of epigraph $E(\psi)$, which is defined as

$$
E(\psi)=\{(u, \alpha) \mid u \in D, \alpha \in \mathbb{R}, \psi(u) \leqslant \alpha\} .
$$

Theorem 3.8. A function $\psi: \mathrm{D} \rightarrow \mathbb{R}$ is a geodesic sub-b-s-convex function with respect to $\mathrm{b}: \mathrm{D} \times \mathrm{D} \times[0,1] \rightarrow \mathbb{R}$ if and only if $\mathrm{E}(\psi)$ is a geodesic sub-b-s-convex set with respect to $\mathrm{b}$.

Proof. Let $(u, \alpha),(v, \beta) \in E(\psi)$. Then

$$
\psi(u) \leqslant \alpha, \quad \psi(v) \leqslant \beta .
$$

Since $\psi$ is geodesic sub-b-s-convex function with respect to $b$ for all $u, v \in D, t \in[0,1]$ and some fixed $s \in(0,1]$, we have

$$
\psi\left(\gamma_{u v}(t)\right) \leqslant t^{s} \psi(u)+(1-t)^{s} \psi(v)+b(u, v, t) \leqslant t^{s} \alpha+(1-t)^{s} \beta+b(u, v, t) .
$$

From the above inequality, it is easy to see that

$$
\left(\gamma_{u v}(t),(1-t)^{s} \beta+t^{s} \alpha+b(u, v, t)\right) \in E(\psi) .
$$

Thus, by Definition 3.7, $\mathrm{E}(\psi)$ is a geodesic sub-b-s-convex set with respect to $b$.

Conversely, if $E(\psi)$ is a geodesic sub-b-s-convex set with respect to $b$ and $u, v \in D$, then

$$
(u, \psi(u)),(v, \psi(v)) \in E(\psi) .
$$

Thus, for all $t \in[0,1]$ and some fixed $s \in(0,1]$, we have

$$
\left(\gamma_{u v}(t),(1-t)^{s} \beta+t^{s} \alpha+b(u, v, t)\right) \in E(\psi) .
$$

This implies that

$$
\psi\left(\gamma_{u v}(t)\right) \leqslant t^{s} \psi(u)+(1-t)^{s} \psi(v)+b(u, v, t),
$$

which shows that $\psi$ is a geodesic sub-b-s-convex function with respect to $b$.

Theorem 3.9. Suppose that $\mathrm{U}_{j}, \mathrm{j} \in \mathrm{J}=\{1, \ldots, \mathrm{m}\}$ is a family of geodesic sub-b-s-convex sets with respect to $\mathrm{b}$. Then their intersection $\cap_{\mathrm{j} \in \mathrm{J}} \mathrm{U}_{\mathrm{j}}$ is also a geodesic sub-b-s-convex set with respect to $\mathrm{b}$.

Proof. Let $(u, \alpha),(v, \beta) \in \cap_{j \in J} u_{j}$. Then for any $j \in J,(u, \alpha),(v, \beta) \in u_{j}$. By using $u_{j}$ being a geodesic sub-b-s-convex set with respect to $b$ for each $j \in J$, it follows that for all $t \in[0,1]$ and some fixed $s \in(0,1]$

$$
\left(\gamma_{u v}(t),(1-t)^{s} \beta+t^{s} \alpha+b(u, v, t)\right) \in u_{j} .
$$

Thus,

$$
\left(\gamma_{u v}(t),(1-t)^{s} \beta+t^{s} \alpha+b(u, v, t)\right) \in \cap_{j \in J} u_{j} .
$$

Therefore, $\cap_{j \in J} U_{j}$ is a geodesic sub-b-s-convex set with respect to $b$.

Theorem 3.10. Let $\mathrm{D} \subset \mathrm{N}$ be a geodesic convex set and $\psi_{j}, j \in J=\{1, \ldots, \mathrm{m}\}$ be a family of real valued functions which are geodesic sub-b-s-convex functions with respect to $\mathrm{b}$ and bounded from above on $\mathrm{D}$. Then function $\psi(\mathfrak{u})=\sup _{\mathbf{j} \in \mathrm{J}} \psi_{\mathbf{j}}(\mathrm{u})$ is a geodesic sub-b-s-convex function with respect to $\mathrm{b}$ on $\mathrm{D}$.

Proof. Since $\psi_{j}$ is a geodesic sub-b-s-convex function with respect to $b$, then its epigraph

$$
E\left(\psi_{j}\right)=\left\{(u, \alpha) \mid u \in D, \alpha \in \mathbb{R}, \psi_{j}(u) \leqslant \alpha, j \in J\right\}
$$

is a geodesic sub-b-s-convex set with respect to $b$. Therefore,

$$
\cap_{j \in J} E\left(\psi_{j}\right)=\left\{(u, \alpha) \mid u \in D, \alpha \in \mathbb{R}, \psi_{j}(u) \leqslant \alpha, j \in J\right\}=\{(u, \alpha) \mid u \in D, \alpha \in \mathbb{R}, \psi(u) \leqslant \alpha\},
$$

where $\psi(u)=\sup _{j \in J} \psi_{j}(u)$. According to Theorem 3.9, we get their intersection is the epigraph of $\psi$. Hence by Theorem 3.8, $\psi$ is a geodesic sub-b-s-convex with respect to $b$. 
We assume now that $\psi$ is geodesic sub-b-s-convex function with respect to $b$ which is continuously differentiable function. For fixed $u, v \in D, b(u, v, t)$ is a continuously decreasing function on $t$. Then, $\frac{b(u, v, t)}{t}$ is a continuously decreasing function about $t$. Moreover, we suppose that the $\lim _{t \rightarrow 0_{+}} \frac{b(u, v, t)}{t}$ exists and the limit is the maximum of $\frac{b(u, v, t)-o(t)}{t}$ for each $t \in(0,1]$ and $u, v \in D$.

Theorem 3.11. Let $\psi: \mathrm{D} \rightarrow \mathbb{R}$ be a non-negative differentiable geodesic sub-b-s-convex function with respect to b. Then

(i) $d \psi_{v}\left(\gamma_{u v}^{\prime}(0)\right) \leqslant t^{s-1}(\psi(u)+\psi(v))+\lim _{t \rightarrow 0_{+}} \frac{b(u, v, t)}{t}$,

(ii) $d \psi_{v}\left(\gamma_{u v}^{\prime}(0)\right) \leqslant t^{s-1}(\psi(u)-\psi(v))+\frac{\psi(v)}{t}+\lim _{t \rightarrow 0_{+}} \frac{b(u, v, t)}{t}$, where $d \psi_{v}$ is the differential of $\psi$ at the point $v$.

Proof.

(i) By the Taylor's expansion of $\psi$, we have

$$
\psi\left(\gamma_{u v}(t)\right)=\psi\left(\gamma_{u v}(0)\right)+t d \psi_{\gamma_{u v}(0)}\left(\gamma_{u v}^{\prime}(0)\right)+o(t) .
$$

Since $\gamma_{u v}(0)=v$, therefore

$$
\psi\left(\gamma_{u v}(t)\right)=\psi(v)+t d \psi_{v}\left(\gamma_{u v}^{\prime}(0)\right)+o(t) .
$$

By the geodesic sub-b-s-convexity of $\psi$ with respect to $b$,

$$
\psi\left(\gamma_{u v}(t)\right) \leqslant t^{s} \psi(u)+(1-t)^{s} \psi(v)+b(u, v, t) .
$$

As $(1-t)^{s} \leqslant\left(1+t^{s}\right)$,

$$
\psi\left(\gamma_{u v}(t)\right) \leqslant t^{s} \psi(u)+\left(1+t^{s}\right) \psi(v)+b(u, v, t) .
$$

The inequalities (3.1) and (3.3) yield

$$
\begin{aligned}
\psi(v)+\mathrm{td} \psi_{v}\left(\gamma_{u v}^{\prime}(0)\right)+o(t) & \leqslant t^{s} \psi(u)+\left(1+t^{s}\right) \psi(v)+b(u, v, t) \\
& \leqslant \psi(v)+t^{s}(\psi(u)+\psi(v))+b(u, v, t),
\end{aligned}
$$

or

$$
\operatorname{td} \psi_{v}\left(\gamma_{u v}^{\prime}(0)\right)+o(t) \leqslant t^{s}(\psi(u)+\psi(v))+b(u, v, t)
$$

On dividing the above inequality by $t$ and using the fact that $\lim _{t \rightarrow 0^{+}} \frac{b(u, v, t)}{t}$ is maximum of $\frac{b(u, v, t)}{t}-$ $\frac{o(t)}{t}$, we have

$$
d \psi_{v}\left(\gamma_{u v}^{\prime}(0)\right) \leqslant t^{s-1}(\psi(u)+\psi(v))+\lim _{t \rightarrow 0^{+}} \frac{b(u, v, t)}{t} .
$$

(ii) Combining the inequalities (3.1) and (3.2), we obtain

$$
\begin{aligned}
\psi(v)+t d \psi_{v}\left(\gamma_{u v}^{\prime}(0)\right)+o(t) & \leqslant t^{s} \psi(u)+(1-t)^{s} \psi(v)+b(u, v, t) \\
& =t^{s} \psi(u)+(1-t)^{s} \psi(v)-t^{s} \psi(v)+t^{s} \psi(v)+b(u, v, t) \\
& \leqslant t^{s}(\psi(u)-\psi(v))+b(u, v, t)+\psi(v)\left((1-t)^{s}+t^{s}\right) .
\end{aligned}
$$

Obviously, $\left((1-t)^{s}+t^{s}\right) \leqslant 2$ for all $t \in[0,1]$ and some fixed $s \in(0,1]$. Using the fact that $\psi$ is a non-negative function, inequality (3.4) reduces to

$$
\psi(v)+\mathrm{td} \psi_{v}\left(\gamma_{u v}^{\prime}(0)\right)+o(t) \leqslant t^{s}(\psi(u)-\psi(v))+b(u, v, t)+2 \psi(v),
$$

or

$$
\operatorname{td} \psi_{v}\left(\gamma_{u v}^{\prime}(0)\right)+o(t) \leqslant t^{s}(\psi(u)-\psi(v))+b(u, v, t)+\psi(v)
$$


By dividing above inequality by $t$ and similarly using the fact $\lim _{t \rightarrow 0^{+}} \frac{b(u, v, t)}{t}$ is maximum of $\frac{b(u, v, t)}{t}-$ $\frac{o(t)}{t}$, we get

$$
\mathrm{d} \psi_{v}\left(\gamma_{\mathfrak{u} v}^{\prime}(0)\right) \leqslant \mathrm{t}^{s-1}(\psi(\mathrm{u})-\psi(v))+\frac{\psi(v)}{\mathrm{t}}+\lim _{\mathrm{t} \rightarrow 0_{+}} \frac{\mathrm{b}(\mathrm{u}, v, \mathrm{t})}{\mathrm{t}} .
$$

Hence the proof is completed.

Theorem 3.12. Let $\psi: \mathrm{D} \rightarrow \mathbb{R}$ be a negative differentiable geodesic sub-b-s-convex function with respect to map $\mathrm{b}(\mathrm{u}, v, \mathrm{t})$. Then

$$
d \psi_{v}\left(\gamma_{u v}^{\prime}(0)\right) \leqslant t^{s-1}(\psi(u)-\psi(v))+\lim _{t \rightarrow 0_{+}} \frac{b(u, v, t)}{t} .
$$

Proof. By the Taylor's expansion and the geodesic sub-b-s-convexity of $\psi$, we have

$$
\begin{aligned}
& \psi\left(\gamma_{u v}(t)\right)=\psi\left(\gamma_{u v}(0)\right)+t d \psi_{\gamma_{u v}(0)}\left(\gamma_{u v}^{\prime}(0)\right)+o(t), \\
& \psi\left(\gamma_{u v}(t)\right) \leqslant t^{s} \psi(u)+(1-t)^{s} \psi(v)+b(u, v, t) .
\end{aligned}
$$

Since for all $t \in[0,1]$ and some fixed $s \in(0,1]$, we have $\left(1-t^{s}\right) \leqslant(1-t)^{s}$. Therefore, inequality (3.6) reduces to

$$
\psi\left(\gamma_{u v}(t)\right) \leqslant t^{s} \psi(u)+\left(1-t^{s}\right) \psi(v)+b(u, v, t) .
$$

The inequalities (3.5) and (3.7) along with $\gamma_{u v}(0)=v$ gives

$$
\mathrm{td} \psi_{\gamma_{u v}(0)}\left(\gamma_{u v}^{\prime}(0)\right)+o(t) \leqslant t^{s}(\psi(u)-\psi(v))+b(u, v, t)
$$

Dividing the above inequality by $t$ and using the fact that $\lim _{t \rightarrow 0^{+}} \frac{b(u, v, t)}{t}$ is maximum of $\frac{b(u, v, t)}{t}-\frac{o(t)}{t}$, it reduces to

$$
d \psi_{v}\left(\gamma_{u v}^{\prime}(0)\right) \leqslant t^{s-1}(\psi(u)-\psi(v))+\lim _{t \rightarrow 0_{+}} \frac{b(u, v, t)}{t} .
$$

Corollary 3.13. Suppose that $\psi: \mathrm{D} \rightarrow \mathbb{R}$ is a differentiable geodesic sub-b-s-convex function with respect to map b. For $\mathrm{t} \in(0,1]$ and $\gamma_{v \mathfrak{u}}^{\prime}(0)=-\gamma_{\mathfrak{u} v}^{\prime}(0)$,

(i) if $\psi$ is a non-negative function, then

$$
\left(d \psi_{v}-d \psi_{u}\right)\left(\gamma_{u v}^{\prime}(0)\right) \leqslant \frac{\psi(v)}{t}+\frac{\psi(u)}{t}+\lim _{t \rightarrow 0^{+}} \frac{b(u, v, t)}{t}+\lim _{t \rightarrow 0^{+}} \frac{b(v, u, t)}{t} ;
$$

(ii) if $\psi$ is negative function, then

$$
\left(d \psi_{v}-d \psi_{u}\right)\left(\gamma_{u v}^{\prime}(0)\right) \leqslant \lim _{t \rightarrow 0^{+}} \frac{b(u, v, t)}{t}+\lim _{t \rightarrow 0^{+}} \frac{b(v, u, t)}{t} .
$$

Proof.

(i) Since $\psi$ is non-negative function, we have

$$
\mathrm{d} \psi_{v}\left(\gamma_{\mathfrak{u} v}^{\prime}(0)\right) \leqslant \mathrm{t}^{s-1}(\psi(u)-\psi(v))+\frac{\psi(v)}{\mathrm{t}}+\lim _{\mathrm{t} \rightarrow 0_{+}} \frac{\mathrm{b}(\mathrm{u}, v, \mathrm{t})}{\mathrm{t}} .
$$

Interchanging $u$ and $v$ in the above inequality, it reduces to

$$
\mathrm{d} \psi_{\mathfrak{u}}\left(\gamma_{v \mathfrak{u}}^{\prime}(0)\right) \leqslant \mathrm{t}^{s-1}(\psi(v)-\psi(u))+\frac{\psi(u)}{\mathrm{t}}+\lim _{\mathrm{t} \rightarrow 0_{+}} \frac{\mathrm{b}(v, \mathrm{u}, \mathrm{t})}{\mathrm{t}} .
$$

Adding inequalities (3.8) and (3.9) and using $\gamma_{v \mathfrak{u}}^{\prime}(0)=-\gamma_{\mathfrak{u} v}^{\prime}(0)$, we obtain

$$
\left(d \psi_{v}-d \psi_{u}\right)\left(\gamma_{u v}^{\prime}(0)\right) \leqslant \frac{\psi(v)}{t}+\frac{\psi(u)}{t}+\lim _{t \rightarrow 0^{+}} \frac{b(u, v, t)}{t}+\lim _{t \rightarrow 0^{+}} \frac{b(v, u, t)}{t} .
$$

(ii) Similarly, if $\psi$ is a negative function, we get

$$
\left(d \psi_{v}-d \psi_{u}\right)\left(\gamma_{u v}^{\prime}(0)\right) \leqslant \lim _{t \rightarrow 0^{+}} \frac{b(u, v, t)}{t}+\lim _{t \rightarrow 0^{+}} \frac{b(v, u, t)}{t} .
$$

The proof is completed. 


\section{Optimality conditions}

In this section, we use the above results to obtain the solution of non-linear programming problems.

Now we consider the unconstrained problem:

$$
\operatorname{Min}\{\psi(u), u \in D\} .
$$

Theorem 4.1. Let $\psi: \mathrm{D} \rightarrow \mathbb{R}$ be a negative differentiable geodesic sub-b-s-convex function with respect to $\mathrm{b}$. If $\mathrm{u}^{\star} \in \mathrm{D}$ and the inequality

$$
\mathrm{d} \psi_{\mathbf{u}^{\star}}\left(\gamma_{\mathbf{u} u^{\star}}(0)\right) \geqslant \lim _{\mathbf{t} \rightarrow 0^{+}} \frac{b(u, v, t)}{t}
$$

holds for all $\mathrm{u} \in \mathrm{D}, \mathrm{t} \in(0,1]$ and some fixed $\mathrm{s} \in(0,1]$, then $\mathrm{u}^{\star}$ is the optimal solution to the problem (P).

Proof. Since $\psi$ is a negative differentiable geodesic sub-b-s-convex function with respect to $b$, then according to Theorem 3.12, we have

$$
d \psi_{v}\left(\gamma_{u v}^{\prime}(0)\right) \leqslant t^{s-1}(\psi(u)-\psi(v))+\lim _{t \rightarrow 0_{+}} \frac{b(u, v, t)}{t}
$$

holds for each $u \in D, t \in(0,1]$ and some fixed $s \in(0,1]$.

On the other hand, we get

$$
\mathrm{d} \psi_{\mathbf{u}^{\star}}\left(\gamma_{\mathbf{u} u^{\star}}(0)\right) \geqslant \lim _{\mathrm{t} \rightarrow 0^{+}} \frac{\mathrm{b}(\mathrm{u}, v, \mathrm{t})}{\mathrm{t}} .
$$

Using above relation in inequality (4.2), we obtain

$$
t^{s-1}\left(\psi(u)-\psi\left(u^{\star}\right)\right) \geqslant 0,
$$

i.e.,

$$
\psi(u)-\psi\left(u^{\star}\right) \geqslant 0,
$$

which implies $u^{\star}$ is the optimal solution to the problem $(\mathrm{P})$.

Theorem 4.2. Suppose that $\psi: \mathrm{D} \rightarrow \mathbb{R}$ is a strictly negative differentiable geodesic sub-b-s-convex function with respect to $\mathrm{b}$. If $\mathrm{u}^{\star} \in \mathrm{D}$ satisfies the condition (4.1), then $\mathrm{u}^{\star}$ is the unique optimal solution to the problem (P).

Proof. Since $\psi$ is strictly negative geodesic sub-b-s-convex function with respect to $b$, then Theorem 3.12 is reduced to

$$
d \psi_{v}\left(\gamma_{u v}^{\prime}(0)\right)<t^{s-1}(\psi(u)-\psi(v))+\lim _{t \rightarrow 0_{+}} \frac{b(u, v, t)}{t} .
$$

Suppose $\mathfrak{u}_{1}, \mathfrak{u}_{2} \in \mathrm{D}$ are two different optimal solution of problem (P). Without loss of generality, we can assume that

$$
\psi\left(u_{1}\right)=\psi\left(u_{2}\right) .
$$

Now, for all $u_{1}, u_{2} \in D, t \in(0,1]$ and for some fixed $s \in(0,1]$, the inequality (4.3) can also be written as

$$
d \psi_{\mathfrak{u}_{2}}\left(\gamma_{\mathfrak{u}_{1} \mathfrak{u}_{2}}(0)\right)-\lim _{\mathrm{t} \rightarrow 0^{+}} \frac{\mathrm{b}\left(\mathfrak{u}_{1}, \mathfrak{u}_{2}, \mathrm{t}\right)}{\mathrm{t}}<\mathrm{t}^{s-1}\left(\psi\left(\mathfrak{u}_{1}\right)-\psi\left(\mathfrak{u}_{2}\right)\right),
$$

hence from inequality (4.1), it yields

$$
t^{s-1}\left(\psi\left(u_{1}\right)-\psi\left(u_{2}\right)\right)>0
$$

that is,

$$
\psi\left(u_{1}\right)-\psi\left(u_{2}\right)>0,
$$

which contradicts to (4.4). This completes the proof . 
We now derive the sufficient optimality conditions for the following constrained programming problem:

$$
\operatorname{Min}\left\{\psi(u) \mid u \in D, g_{j}(u) \leqslant 0, j \in J\right\}, \quad J=\{1,2, \ldots, m\} .
$$

Let $\mathrm{S}$ denote the set of all feasible solution to the problem $\left(\mathrm{P}_{\mathrm{s}}\right)$, i.e.,

$$
S=\left\{u \in D \mid g_{j}(u) \leqslant 0, j \in J\right\} .
$$

Theorem 4.3 (Karush-Kuhn-Tucker sufficient optimality conditions). Let $\psi: \mathrm{D} \rightarrow \mathbb{R}$ be a negative differentiable geodesic sub-b-s-convex function with respect to $\mathrm{b}: \mathrm{D} \times \mathrm{D} \times[0,1] \rightarrow \mathbb{R}$ and $\mathrm{g}_{\mathrm{j}}: \mathrm{D} \rightarrow \mathbb{R}(j \in \mathrm{J})$ be differentiable sub-b-s-convex function with respect to $b_{j}$. Suppose that $\bar{u} \in S$ is a KKT point of $\left(\mathrm{P}_{\mathrm{s}}\right)$, that is, there exist multipliers $\lambda_{j} \geqslant 0(j \in J)$ such that

$$
\mathrm{d} \psi_{\bar{u}}+\sum_{j \in J} \lambda_{j} d g_{j \bar{u}}=0, \lambda_{j} g_{j}(\bar{u})=0
$$

If

$$
\lim _{t \rightarrow o^{+}} \frac{b(u, \bar{u}, t)}{t} \leqslant-\sum_{j \in J} \lambda_{j} \lim _{t \rightarrow 0^{+}} \frac{b_{j}(u, \bar{u}, t)}{t},
$$

then $\overline{\mathrm{u}}$ is an optimal solution to the problem $\left(\mathrm{P}_{\mathrm{s}}\right)$.

Proof. For any $u \in S$, we have

$$
g_{j}(u) \leqslant 0=g_{j}(\bar{u}), j \in J(\bar{u})=\left\{j \in J \mid g_{j}(\bar{u})=0\right\} .
$$

By the geodesic sub-b-s-convexity of $g_{j}$ with respect to $b_{j}$ and the Theorem 3.12 , for $j \in J(\bar{u})$, we obtain

$$
\operatorname{dg}_{j \bar{u}}\left(\gamma_{u \bar{u}}^{\prime}(0)\right)-\lim _{t \rightarrow 0^{+}} \frac{b(u, \bar{u}, t)}{t} \leqslant t^{s-1}\left(g_{j}(u)-g_{j}(\bar{u})\right) \leqslant 0 .
$$

From inequality (4.5), we get

$$
d \psi_{\bar{u}}\left(\gamma_{u \bar{u}}^{\prime}(0)\right)=-\sum_{j \in J} \lambda_{j} d g_{j \bar{u}}\left(\gamma_{u \bar{u}}^{\prime}(0)\right)=-\sum_{j \in J(\bar{u})} \lambda_{j} d g_{j \bar{u}}\left(\gamma_{u \bar{u}}^{\prime}(0)\right) .
$$

The above equation along with inequality (4.6) gives

$$
\begin{aligned}
d \psi_{\bar{u}}\left(\gamma_{u \bar{u}}^{\prime}(0)\right)-\lim _{t \rightarrow 0^{+}} \frac{b(u, \bar{u}, t)}{t} & \geqslant-\sum_{j \in J} \lambda_{j} d g_{j \bar{u}}\left(\gamma_{u \bar{u}}^{\prime}(0)\right)+\sum_{j \in J} \lambda_{j} \lim _{t \rightarrow 0^{+}} \frac{b(u, \bar{u}, t)}{t} \\
& =-\sum_{j \in J(\bar{u})} \lambda_{j}\left(d g_{j \bar{u}}\left(\gamma_{u \bar{u}}^{\prime}(0)\right)-\lim _{t \rightarrow 0^{+}} \frac{b(u, \bar{u}, t)}{t}\right) .
\end{aligned}
$$

Combining the inequality (4.7) with the above inequality, yields

$$
\mathrm{d} \psi_{\overline{\mathrm{u}}}\left(\gamma_{\mathrm{u} \overline{\mathrm{u}}}^{\prime}(0)\right)-\lim _{\mathrm{t} \rightarrow 0^{+}} \frac{\mathrm{b}(\mathrm{u}, \overline{\mathrm{u}}, \mathrm{t})}{\mathrm{t}} \geqslant 0 .
$$

From Theorem 4.1, it becomes

$$
\psi(\mathfrak{u})-\psi(\bar{u}) \geqslant 0
$$

for each $u \in S$. Hence $\bar{u}$ is an optimal solution to the problem $\left(P_{S}\right)$. 


\section{Conclusion}

In this paper, we have introduced a new class of functions and sets, called geodesic sub-b-s-convex function and geodesic sub-b-s-convex set, and studied their properties for general and differentiable cases. Furthermore, the optimality conditions for a non-linear programming problem are also derived. We can also study duality results of aforesaid class of functions, which builds the future work of authors.

\section{Acknowledgment}

The research of the first author is financially supported by King Fahd University of Petroleum and Minerals, Saudi Arabia under the Internal Project No. IN161058.

\section{References}

[1] R. P. Agarwal, I. Ahmad, A. Iqbal, S. Ali, Generalized invex sets and preinvex functions on Riemannian manifolds, Taiwanese J. Math., 16 (2012), 1719-1732. 1

[2] I. Ahmad, A. Iqbal, S. Ali, On properties of geodesic ๆ-preinvex functions, Adv. Oper. Res., 2009 (2009), 10 pages. 1

[3] T. Antczak, r-preinvexity and r-invexity in mathematical programming, Comput. Math. Appl., 50 (2005), 551-566. 1

[4] A. Barani, M. R. Pouryayevali, Invex sets and preinvex functions on Riemannian manifolds, J. Math. Anal. Appl., 328 (2007), 767-779. 1

[5] M. T. Chao, J. B. Jian, D. Y. Liang, Sub-b-convex functions and sub-b-convex programming, Oper. Res. Trans., 16 (2012), 1-8. 1

[6] M. A. Hanson, On sufficiency of the Kuhn-Tucker conditions, J. Math. Anal. Appl., 80 (1981), 545-550. 1

[7] H. Hudzik, L. Maligranda, Some remarks on s-convex functions, Aequationes Math., 48 (1994), 100-111. 1

[8] A. Iqbal, I. Ahmad, S. Ali, Strong geodesic $\alpha$-preinvexity and invariant $\alpha$-monotonicity on Riemannian manifolds, Numer. Funct. Anal. Optim., 31 (2010), 1342-1361. 1

[9] A. Iqbal, I. Ahmad, S. Ali, Some properties of geodesic semi-E-convex functions, Nonlinear Anal., 74 (2011), $6805-6813$. 1

[10] A. Iqbal, S. Ali, I. Ahmad, On geodesic E-convex sets, geodesic E-convex functions and E-epigraph, J. Optim. Theory Appl., 155 (2012), 239-251. 1

[11] A. Kiliçman, W. Saleh, On geodesic strongly E-convex sets and geodesic strongly E-convex functions, J. Inequal. Appl., 2015 (2015), 10 pages. 1

[12] J. Liao, T. Du, On some characterization of sub-b-s-convex functions, Filomat, 30 (2016), 3885-3895. 1, 3

[13] T. Rapcsák, Geodesic convexity in nonlinear optimization, J. Optim. Theory Appl., 69 (1991), 169-183. 1

[14] C. Udrişte, Convex functions and optimization methods on Riemannian manifolds, Kluwer Academic Publishers, Dordrecht, (1994). 1, 2.1, 2.2

[15] E. A. Youness, On E-convex sets, E-convex functions and E-convex programming, J. Optim. Theory Appl., 102 (1999), 439-450. 1

[16] L.-W. Zhou, N.-J. Huang, Roughly geodesic B-invex and optimization problem on Hadamard manifolds, Taiwanese J. Math., 17 (2013), 833-855. 1 